Yokoigawa, Miki.

Profesor Investigador Titular, Universidad Autónoma del Estado de Hidalgo, Área Académica de Artes Visuales, Cuerpo AcadémicoPráctica Visual en el Arte Actual (Laboratorio AV).

\title{
Para posible conservación de la memoria de la catástrofe en el estudio del Tourizing Fukushima: The Fukuichi Kanko Project.
}

\author{
TIPO DE TRABAJO \\ Comunicación.
}

PALABRAS CLAVE

Memoria, archivo, art activism.

KEY WORDS

Memory, archive, art activism.

\section{RESUMEN}

Después de cuatro años de ocurrida la catástrofe del terremoto, el tsunami y el accidente de las plantas nucleares de Fukushima en Japón, hay ciertas preocupaciones en torno a la reconstrucción de la sociedad posFukushima y la inquietud por el olvido de dicho suceso, es decir, la memoria de la catástrofe.

En ese contexto, encontramos un planteamiento radical: Tourizing Fukushima: The Fukuichi Kanko Project, el cual consiste en convertir Fukushima en un lugar turístico con interés comercial. Éste es un proyecto bastante polémico pues ha provocado malestar y desacuerdo por el concepto turístico. El grupo colectivo presentó el proyecto en dos publicaciones: Guía de Chernobyl Dark Tourism, y la segunda, Tourizing Fukushima: The Fukuichi Kanko Project. En ambas hay una propuesta sobre la conservación de la memoria a través de los archivos y su nexo con la realidad virtual. Este proyecto tiene un aspecto artístico por el cambio de perspectiva para aproximarnos a la realidad por medio de la tecnología virtual.

\section{ABSTRACT}

After four years of the disaster of the earthquake, the tsunami and the accident at the nuclear plant in Fukushima in Japan, there are certain concerns about rebuilding the posFukushima society and concern about the forgetfulness of the event, that is to say, the memory of the catastrophe.

In this context, we find a radical approach: Tourizing Fukushima: The Fukuichi Kanko Project, which is to turn Fukushima into a tourist spot with commercial interests. This is a rather controversial project because it has caused discomfort and disagreement with the tourist concept. The collective group presented the project in two publications: Dark Tourism Guide Chernobyl, and the second, Tourizing Fukushima: The Fukuichi Kanko Project. In both there is a proposal on the retention of memory through the files and its connection with virtual reality. This project has an artistic look for change of perspective to approach reality through virtual technology. 


\section{CONTENIDO}

\section{INTRODUCCIÓN}

Después de los eventos ocurridos en Fukushima el 11 de marzo de 2011 se ha suscitado un conflicto entre los sectores públicos y privados. En la zona afectada se planteó la conservación de los "restos del desastre", discutiendo la importancia de una didáctica a partir de la memoria frente a la resistencia del olvido, mientras que diferentes sectores sociales comenzaron la inmediata reconstrucción de la sociedad posdesastre. Por ejemplo, la asociación de arquitectos $\operatorname{ArchiAid}^{1}$ ha planteado el rediseño urbanopopular de esta área, evitando la construcción uniforme que exige la lógica tecnocrática, la cual no resuelve el problema local, además de demostrar su fragilidad frente al desastre natural. Por otro lado, dada la dificultad del problema que significó el accidente nuclear de Fukushima, se está evitando ofrecer soluciones al problema, dejando en el olvido a alrededor de 71399 personas refugiadas ${ }^{2}$ y una cantidad inmensa de materiales contaminados por la radiación.

\section{DESARROLLO}

En torno del accidente de la central nuclear de Fukushima, el grupo interdisciplinar ${ }^{3}$ Fukushima Daiichigenpatsu Kankoukakeikaku Seisaku linkai (FDKSI) propuso en Genron ${ }^{4}$ un proyecto: convertir a Fukushima en un lugar turístico con cierto interés comercial, basándose para ello en el estudio de Dark Tourism de Chernobyl. ${ }^{5}$

En forma concreta, con el proyecto Tourizing Fukushima: The Fukuichi Kanko Project, FDKSI propone organizar una exposición acerca de los juegos olímpicos de 2020, rentabilizando el desastre y ocupando para ello las instalaciones del J-Village. ${ }^{6}$ Para ello es necesario construir un museo o Hazard Center en Tokio con la misma temática y disponer transporte entre Fukushima y Tokio con el fin de alinear Asia-Tokio-Fukushima para, posteriormente, en el 2036, crear el Fukushima Gate Village, rehabilitando el J-Village como un centro de visitantes que cumpla la función de centro académico, comercial y museo, y hacer de dicho espacio un punto de partida para visitar las plantas nucleares y conocer el proceso de desmontaje.

Este proyecto, encabezado por Hiroki Azuma, ${ }^{7}$ comenzó en 2012 en una mesa redonda que formó parte de la exposición colectiva "Hikkurikaeru-ten (Turning around)", 8 con sede en el museo Watari-Um, con la participación de artistas que realizan art activism como Chin $\uparrow$ pom, ${ }^{9}$ de Japón; JR, de Francia; Voina, de Rusia; Adbusters, de Canadá, y otros más. Dentro de la exposición se encontraban ciertas expresiones relacionadas con el suceso de Fukushima, y a partir del evento mencionado Azuma organiza FDKSI.

Ahora bien, el dark tourism (turismo oscuro) comenzó a ser investigado en los noventa en Europa. Comenzaron a llamarse dark tourism a partir de la publicación Dark Tourism: The Attraction of Death and Disaster, ${ }^{10}$ que ha definido estas actividades como las visitas a monumentos de guerra o ruinas de desastres naturales. Los campos de exterminio usados durante la Segunda Guerra Mundial; Chernobyl, el accidente nuclear de 1986 en Ucrania; Grand Zero en Nueva York en 2001 (en EeUu); Banda Aceh y el tsunami de 2004 en Indonesia son puntos bastante reconocidos de turismo oscuro.

\footnotetext{
${ }^{1}$ ArchiAid: Relief and Recovery by Architects for Tohoku Earthquake and Tsunami [http://archiaid.org/english/]. [18 de abril de 2015].

2 De los refugiados de Fukushima, más de la mitad (casi 46902 personas) están en otra prefectura. El dato fue citado por el último reporte sobre el número de refugiados con fecha del 31 de marzo de 2015 de la Reconstrucción Agency del gobierno japonés [http://www.reconstruction.go.jp/topics/main-cat2/sub-cat2-1/20150331_hinansha.pdf]. [21 de abril de 2015].

${ }^{3}$ El comité está formado por ocho investigadores: Akira Ide, del Estúdio del turismo; Kazuki Umezawa, artista visual; Hiroshi Kainuma, sociólogo; Ryo Shimizu, programador; Daisuke Tsuda, periodista y media activista; Kenrou Hayamizu, escritor y editor independiente; Ryuji Fujimura, arquitecto, y Hiroki Azuma. Hay observadores que colaboran en el desarrollo del proyecto, como Yoko Ueda, traductora e investigadora de literatura y teatro ruso; Kebshu Shintsubo, fotógrafo; Hidenori Watanabe, arquitecto e informático, y otros más.

${ }^{4}$ Genron [http://genron.co.jp/]. [15 de abril de 2015].

${ }^{5}$ Dicho proyecto se dio a conocer en dos tomos: Chernobyl Dark Tourism Guide (Guía de Chernobyl Dark Tourism). En Shiso Chizu beta, vol. 4-1, Genron, Tokio, 2013; y Fukushima Daiichiguempatsu kankoukakeikaku gaido Tourizing Fukushima: The Fukuichi Kanko Project (El proyecto turístico del Primer centro nuclear de Fukushima). En Shiso Chizu beta, vol. 4-2, Genron, Tokio, 2013.

${ }^{6} \mathrm{~J}$-Village es un centro deportivo asignado al equipo nacional de fútbol, fue donado por Tokyo Electric Power Company (TEPCO) para promoción regional en 1996. Actualmente funciona como base de TEPCO para el tratamiento del accidente.

${ }^{7}$ Hiroki Azuma, nacido en Tokio en 1971, es el fundador de Genron y uno de los críticos más influyentes de Japón. Sus publicaciones en inglés incluyen el libro sobre el pensamiento político Otaku: Japan's Database Animals. University of Minnesota Press, 2007; y Ontological, Postal, a Book on French Modern Philosophy. 1998, la cual recibió el Premio Suntory para las Ciencias Sociales y Humanidades; también publicó General Will 2.0. Vertical, Nueva York, 2014. Su obra de ciencia ficción Quantum (2009) recibió el Premio $23^{\circ}$ de Yukio Mishima. Sus tweets pueden leerse en @hazuma en japonés y @hazuma_en en inglés.

8 "Hikkurikaeru-ten (Turning around)", del 1 de abril al 29 de julio de 2012, en Watari-Um, The Watari Museum of Contemporary Art [http://www.watarium.co.jp/exhibition/1204hikkuri/index.html]. [15 de abril de 2015].

${ }^{9}$ Chin $\uparrow$ pom es un grupo de seis jóvenes artistas japoneses. Por el accidente nuclear de Fukushima realizaron intervenciones al mural de Taro Okamoto que se encuentra en Shibuya, Tokio, añadiendo una escena de Fukushima con el tema de la energía nuclear, así visualizaron la continuidad histórica del asunto. Para más detalles véase el artículo: Yokoigawa, Miki: "Real Times: art-activismo en tiempo real de posFukushima en Japón”, en Memoria del V Encuentro de Investigación y Documentación de Artes Visuales. Inventar el futuro. Constricción Política y Acción Cultural. Cenidiap-INBA, México, 2015, pp. 243-251.

${ }^{10}$ Lennon, John y Foley, Malcolm: Dark Tourism: The Attraction of Death and Disaster, Cengage Learning EMEA, 2000.
} 
El accidente de la central nuclear de Chernobyl, primera experiencia para la humanidad de un desastre de esta magnitud, tiene 29 años de ocurrido y todavía no ha comenzado el proceso de deshacer los reactores. A partir de 2010 el gobierno ucraniano permitió el acceso a la central nuclear y a la zona de exclusión para realizar visitas guiadas exclusivamente. Antes de la autorización gubernamental, también existían visitas a la zona y los ex habitantes acompañaban a los turistas para facilitar el trámite de acceso e informar sobre el suceso, las víctimas y la experiencia de la diáspora. Actualmente, en Fukushima los ex habitantes se han convertido en guías voluntarios, sin que esto se considere una actividad comercial ni turística; por ello FDKSI argumenta que debe ser una actividad profesional que remunere, lo que mejoraría la calidad de esta labor basándose en un conocimiento global.

Akira Ide, investigador de este tipo de turismo y miembro del FDKSI, insiste en la validez de dicho turismo para la conservación de la memoria traumática en el lugar del suceso; en su artículo "Pensar a partir de Dark Toursim" ${ }^{11}$ argumenta la necesidad del turismo oscuro como una actividad de sucesión de la aflicción de la zona con el sentimiento de las víctimas, esperando el efecto de catarsis. Ide presenta el caso de Banda Aceh, ${ }^{12}$ que ha logrado superar el estado traumático conservando la memoria triste y el escarmiento del desastre natural a través del turismo activo, a partir de los restos conservados del desastre, lo que ha detonado una importante actividad comercial en la zona. Los visitantes reciben información en un museo y el impacto directo se da a través del contacto con los restos, por lo que comprenden el suceso y además experimentan compasión por las víctimas y sus seres queridos. Por otra parte, el proceso de reconstrucción en sí se convierte en la identidad de la zona y los visitantes comparten esa identidad al observar el proceso. Por el contrario, en el caso de la mina Konomai de Monbetsu, en Japón, se está dificultando la sucesión de la memoria por no poder acceder a los restos, pues tan sólo está un monumento de piedra. Por no tener acceso a los restos, además de otras dificultades que no favorecen el turismo, prácticamente no llega nadie, no se genera ninguna actividad económica y no hay actualización de la sucesión; su triste memoria ha quedado encerrada.

Además de la conservación del espacio y el objeto físico que evoca el suceso, la conservación de la memoria puede hacer uso de la realidad virtual para comprender la relación y el vínculo de cada asunto y facilitar el acceso al open archive, lo que posibilita considerar el suceso desde distintas dimensiones. En el proyecto The East Japan Earthquake Archive (EJEA), ${ }^{13}$ que inició el 24 de marzo de 2011 , se comenzó a facilitar la comunicación de la realidad compleja del desastre y del accidente ${ }^{14}$ a través de una plataforma digital. Hidenori Watanabe, observador del FDKSI, ganó el premio Ars Electronica $2013^{15}$ por organizar EJEA con su colectivo, cuyo antecedente fue la construcción de Nagasaki Archive, ${ }^{16}$ presentado en 2010, e Hiroshima Archive, ${ }^{17}$ en 2011, los cuales cimentaron la base de una plataforma digital con mapping en imágenes 3D a través de Google Earth. EJEA posibilita reflexionar la imposibilidad de unificar y uniformar las imágenes del suceso, además de los testimonios y múltiples puntos de vista. Watanabe argumenta que el EJEA sería una herramienta que permitiría visibilizar el carácter polifacético de la imagen para ir más allá del estereotipo de Fukushima: accidente nuclear, ruinas contaminadas, como sucedió con el videojuego S.T.A.L.K.E.R., ${ }^{18}$ en el que se exageran los elementos. Pero esta interfaz, como cualquier otra tecnología, tiene una durabilidad limitada, de allí que la conservación de la memoria, para superar este problema, requiera que las personas continúen narrando el suceso. En el caso de Hiroshima Archive, las estudiantes de una preparatoria entrevistaron a los ancianos sobrevivientes de la bomba, registraron varios testimonios de quienes guardaron durante 70 años la memoria como secreto. Al escuchar estos testimonios, las jóvenes tuvieron la posibilidad de narrar lo experimentado, siendo primeras usuarias de la interfaz. Estas actividades formaron "la comunidad de la memoria", como la denomina Watanabe: un movimiento que actualiza la sucesión de la memoria activando el Archive. Del mismo modo, en Fukushima se está construyendo "la comunidad de la memoria". Los estudiantes de la zona realizan mapping de los testimonios documentados y los vecinos de la zona comienzan a ofrecer información voluntariamente. Es muy diferente la información reunida en Fukushima de la de Hiroshima; sin embargo, ambos casos comparten asuntos bastante delicados como son la contaminación radiactiva, el daño o la segregación por el rumor.

Watanabe no niega el riesgo de malentendido o la mala interpretación del Archive, por lo que serían necesarias lecciones continuas para fortalecer "la comunidad de la memoria". Actualmente Fukushima tiene una dificultad, por la separación física causada por el accidente, para reconstruir su comunidad, pero existen antecedentes como Virtual Pripyat, ${ }^{19}$ en Chernobyl, y Tomioka Inside, ${ }^{20}$ en

\footnotetext{
${ }^{11}$ Ide, Akira: "Dark Tourism kara kangaeru (Pensar a partir de Dark Tourism)". En Tourizing Fukushima: The Fukuichi Kanko Project (El proyecto turístico del Primer centro nuclear de Fukushima). En Shiso Chizu beta, vol. 4-2, Genron, Tokio, 2013, pp. 144-157.

${ }_{12}$ Banda Aceh Tourism [http://bandaaccehtourism.com/]. [19 de abril de 2015].

${ }^{13}$ The East Japan Earthquake Archive [http://nagasaki.mapping.jp/p/japan-earthquake.html]. [15 de abril de 2015].

${ }^{14}$ Watanabe, Hidenori: "Kiokuo tsutaeru (Comunicar la memoria)". En Tourizing Fukushima: The Fukuichi Kanko Project (El proyecto turístico del Primer centro nuclear de Fukushima). En Shiso Chizu beta, vol. 4-2, Genron, Tokio, 2013, pp. 48-51.

${ }^{15}$ Ars Electronica [http://www.aec.at/news/]. [22 de abril de 2015] ha premiado al The East Japan Earthquake Archive en 2013 [http://archive.aec.at/prix/\#48559] [22 de abril de 2015] y al Proyect Funbaro East Japan en 2014 [http://archive.aec.at/prix/\#48994] [22 de abril de 2015].

${ }^{16}$ Nagasaki Archive [http://nagasaki.mapping.jp/] [22 de abril de 2015].

${ }^{17}$ Hiroshima Archive [http://hiroshima.mapping.jp/] [22 de abril de 2015]

${ }^{18}$ S.T.A.L.K.E.R. [http://www.stalker-game.com/] [22 de abril de 2015].

${ }^{19}$ Virtual Pripyat [http://addyour.name/index.php?lang=en] [22 de abril de 2015].

${ }^{20}$ Tomioka Inside [http://www.tomioka.jpn.org/ [22 de abril de 2015].
} 
Tomioka; comunidades en las que se desarrollan actividades a través de una red virtual civil después de la diáspora. En este sentido la interfaz podría ser una herramienta indispensable para establecer una nueva comunidad.

\section{CONCLUSIÓN}

Hemos observado los primeros pasos para la realización del Tourizing Fukushima: The Fukuichi Kanko Project, cuyo motivo principal es la resistencia social al olvido y la apatía sobre el suceso de Fukushima. El proceso de desmontar los reactores nucleares seguramente tardará unas décadas y poder controlar la contaminación radiactiva necesitará cientos de años, así que la estabilización de la zona requiere mucho tiempo y amplio apoyo.

No somos capaces de juzgar si el proyecto es apto o no, pero este proyecto nos permite visualizar la realidad de Fukushima, que no se reduce a una imagen, sino que tiene un aspecto prolífico, realidad que cambiaría el punto de vista para enfrentarla y generar una red de complicidad entre todos.

Es clara la presencia de la imagen en torno de una catástrofe natural como lo fue el terremoto y tsunami que afectó el noreste de Japón, incluida Fukushima. Pero un proyecto como el anterior trata de visualizar hechos invisibles: la radiación, el sufrimiento, la pérdida y la diáspora de las víctimas. Es necesario ver la imagen acerca del suceso natural en su multiplicidad, no sólo recogida por los medios masivos sino a través de las miles de capas, opiniones y memorias de los afectados; es aquí donde el sistema virtual nos permite romper el estereotipo, citando datos que nos permitan visualizar el hecho pero no la memoria. Por ello este proyecto puede ser una gran narración que simplemente espera ser activada por el espectador que usa la interfaz, el sistema y sus herramientas, para posibilitar una nueva construcción de la memoria, acto que se relaciona ampliamente con el campo artístico.

\section{FUENTES REFERENCIALES}

Bibliografía

Memoria del V Encuentro de Investigación y Documentación de Artes Visuales. Inventar el futuro. Constricción Política y Acción Cultural. Cenidiap-inba, México, 2015

Azuma, Hiroki (ed.): Chernobyl Dark Tourism Guide (Guía de Chernobyl Dark Tourism). En Shiso Chizu beta, vol. 4-1, Genron, Tokio, 2013.

Fukushima Daiichiguempatsu kankoukakeikaku gaido Tourizing Fukushima: The Fukuichi Kanko Project (El proyecto turístico del Primer centro nuclear de Fukushima). En Shiso Chizu beta, vol. 4-2, Genron, Tokio, 2013.

Lennon, John y Foley, Malcolm: Dark Tourism: The Attraction of Death and Disaster, Cengage Learning EMEA, 2000.

Web

Ars Electronica [http://www.aec.at/news/] [22 de abril de 2015].

ArchiAid: Relief and Recovery by Architects for Tohoku Earthquake and Tsunami [http://archiaid.org/english/] [18 de abril de 2015].

Banda Aceh Tourism [http://bandaaccehtourism.com/] [19 de abril de 2015].

Genron [http://genron.co.jp/en] [10 de abril de 2015].

Hiroshima Archive [http://hiroshima.mapping.jp/] [22 de abril de 2015].

Nagasaki Archive [http://nagasaki.mapping.jp/] [22 de abril de 2015].

Proyect Funbaro East Japan (Premio Ars Electronica) [http://archive.aec.at/prix/\#48994] [22 de abril de 2015].

Reporte sobre el número de refugiados con fecha del 31 de marzo de 2015 de Reconstrucción Agency del gobierno japonés [http://www.reconstruction.go.jp/topics/main-cat2/sub-cat2-1/20150331_hinansha.pdf] [21 de abril de 2015]. 
S.T.A.L.K.E.R. [http://www.stalker-game.com/] [22 de abril de 2015].

The East Japan Earthquake Archive [http://nagasaki.mapping.jp/p/japan-earthquake.html] [15 de abril de 2015].

The East Japan Earthquake Archive (Premio Ars Electronica) [http://archive.aec.at/prix/\#48559] [22 de abril de 2015].

Tomioka Inside [http://www.tomioka.jpn.org/] [22 de abril de 2015].

Virtual Pripyat [http://addyour.name/index.php?lang=en] [22 de abril de 2015].

Watari-Um, The Watari Museum of Contemporary Art [http://www.watarium.co.jp/exhibition/1204hikkuri/index.html]. 